\title{
The Endomysium of Human Embryonic Hyoid Muscle during Development
}

\author{
Iwao SATO, Masataka SUNOHARA, Toshiya FUJITA and Toru SATO
}

Department of Anatomy, School of Dentistry at Tokyo, Nippon Dental University, Tokyo 102-8159 Japan

- Received for Publication, August 26, 1998 -

Key Words: Hyoid muscle, Development, Muscle, Endomysium

\begin{abstract}
Summary: A computer analysis was performed on the endomysium of the hyoid muscle in human fetuses, which ranged in gestational week from 12 to 32 weeks. During development of the human hyoid muscle a level of intramuscular connective tissue as reticular fibers displays a specific change in morphology. We examined quantitates and distributions of endomysium in human hyoid muscles from 12 to 32 weeks. The endomysium of the hyoid muscles formed complex structures, and volume rates increased from 20 weeks gestation. On the other hand, the cross sectional area (CSA) of muscle fibers had almost the same profile. The reticular fibers increased during development from 16 to 28 weeks gestation.
\end{abstract}

Collagenous fibrils can be roughly classified into two types, straight parallel fibrils (Fawcett, 1986), and helically arranged, wavy fibrils. The collagenous fibrils form sinuous bundles and three-dimensional sheaths (Rizk, 1980). The organized layers of a muscle are composed of the epimysium, perimysium and endomysium. Endomysium, which separates from each muscle fiber and covers the surface muscle fiber, are called ensheaths (Walls, 1960). The connective tissue networks of these organizations in the skeletal muscle contained extracellular matrices with growth factors (Mayne and Sanderson, 1985). Sato and Shimada (1997) reported that the fibrile collagen of extracellular matrix affects development of masticatory muscles. The extracellular matrix components are related to patterns of muscle fibers (Chevallier et al., 1977; Kieny and Chevallier, 1980). Transforming growth factor beta 1 is implicated in formation of patterns of muscle fibers (McLennan, 1993) in addition to temporal and positional factors (McLennan, 1983; Narusawa et al., 1987).

This framework with its collagen fibrils that surround each muscle fiber is involved in resisting stretching forces during a movement (Schmalbruch, 1974). Vries et al. (1982) reported that rotation of the head movement occurs from 9 week to 13 weeks gestation, and is often associated with retroflexions of the head. Hooker (1952) and Birnholz (1978) also reported that the head movement occurs at an early stage of the fetus. The opening of the jaw occurs from 8.5 weeks (Humphrey, 1968) and sucking and swallowing with drinking amniotic fluid is displayed from 12.5 weeks (Vries et al, 1982). Nishimura et al. (1996) found intramuscular connective tissue of bovine semitendinous muscle decreased during development. Therefore, the morphology of the connective tissue as an endomysium is an important element in function and development of the human hyoid muscles.

In the present study, we examined the quantity of endomysium and the muscle fibers of the hyoid muscles during development in human fetuses, which ranged from 12 to 32 weeks gestation.

\section{Materials and Methods}

Twelve fetuses were processed at autopsy for the immunocytochemical analysis for the morphological analysis of the hyoid muscles (superior hyoid: anterior belly of digastric, posterior belly of digastric, mylohyoid, stylohyoid and geniohyoid; inferior hyoid: superior belly of omohyoid, inferior belly of omohyoid, sternohyoid, thyrohyoid and sternothyroid). The fetuses were obtained from legally approved, induced abortions and spontaneous abortions. After abortion, such investigations are part of the legally required autopsy. Twelve fetuses at autopsy were selected from donations at Nippon Dental University. The approximate fetal age was assigned by reference to Streeter's Tables (Streeter, 1920, 1942, 1948, 1951). These specimens were fixed for a $30 \mathrm{~min}$ in a solution of $10 \%$ paraformalin. After specimens had been washed in 
running water, the hyoid muscles were dehydrated in absolute ethyl alcohol and then embedded in paraffin. Serial frontal sections were made at a thickness of about $3 \mu \mathrm{m}$ on a rotary microtome. Reticular fiber was demonstrated in the intramuscular connective tissue of hyoid muscles using a silver impregnation technique (Sano, 1954) in order to differentiate between the epimysium and perimysium. Sections of muscle fibers were observed under a light microscope (Vanox-S; Olympas Co., Tokyo). The cross sectional area (CSA) of individual muscle fiber $\left(\mu \mathrm{m}^{2}\right)$ in each section were measured with an image analyzer (Pias LA-500, Pias Co., Osaka, Japan) linked to a microcomputer (PC9801 VX, NEC, Tokyo, Japan). Ten areas were selected at random on the CSA of muscle fibers. The measurement areas for the ratio of reticular fiber per $10,000 \mu \mathrm{m}^{2}$ were selected from each section and measured with 400 magnification by a light microscope. The densitometric profiles of the reticular fiber with sliver staining were determined with a color image analyzer (Image Hyper II, Interquest Co., Osaka, Japan) linked to a microcomputer (Dion, Tokyo, Japan; Fig. 1).

\section{Results}

\section{Microscopic observations of the hyoid muscles}

At 12 weeks gestation, large myotube cells were found in the cross section of the hyoid muscles. Reticular fibers (endomysium) with silver staining were found around these large muscle fibers (Fig. 2a). The endomysium showed a honeycomb-like structure surrounding the muscle fibers and the cross section of muscle fiber increased during development. From 16 weeks gestation, collagenous bundles of the perimysium were found around these small muscle fibers (Fig. 2b). The endomysium was made up of irregularly arranged fibers which reacted strongly with sliver staining from 20 weeks (Fig. 2c). The fine reticular fibers were found around muscle fibers which has loose contact with the perimysium fiber bundles. At 28 and 32 weeks, numerous reticular fibers were arranged in irregular patterns and formed a network around muscle fiber (Figs. 2d-f).

\section{Analysis of muscle fiber and reticular fiber with a} computer system

The ratios of the reticular fiber (RF) in each section are shown in Fig. 3a,b. In the earlier stage (12 weeks gestation), the rates of RF with silver staining are almost $15 \%$ in anterior belly of digastric (AD), the stylohyoid (SH), the thyrohyoid (TH), the sternothyroid (ST). The rates of the RF in other hyoid muscles are $10 \%$ below. The percentages of the RF increased gradually in the AD, the posterior belly of digastric (PD), the geniohyoid (GH), the inferior belly of omohyoid (IO), and the sternohyoid (STH) during development. The percentages of the RF are almost constant in the $\mathrm{SH}$, the ST and the TH from 12 to 32 weeks. Although the percentage of the RF increased gradually from 24 weeks, it is constant low, ranging from 12 to 24 weeks in the superior belly of omohyoid (SO). The percentage of the RF increased strikingly from 12 to 20 weeks, and decreased at 24 weeks in the mylohyoid (MH).

\section{The CSA of muscle fiber in hyoid muscle}

In the earlier stage (12 weeks gestation), the CSA of muscle fiber is large in the inferior hyoid muscles as compared with that of the superior hyoid muscles. From 24 weeks gestation, the CSA of muscle fiber slightly increased. In contrast, the CSA of muscle fiber in the GH was still low and was constant, ranging from 12 to 32 weeks. From 12 to 24 weeks the CSA of muscle fiber was still low and increased gradually from 28 weeks in the AD, the $\mathrm{MH}$, and the SH. At the 12 weeks the CSA of muscle fiber was large, and gradually decreased from 16 to 20 weeks, and finally increased at 28 and 32 weeks in the SO, the IO, and the TH. The CSA of muscle fiber gradually decreased from 12 to 20 weeks and was constant low in the PD and the STH. The ST maintained the same value at 12,28 , and 32 weeks, and the value was still lower at 16 and 24 weeks.

\section{Discussion}

\section{The muscle fiber during development}

In the early stage, the myotube fibers are clearly visible and the formation of muscle fibers can be seen at 20 and 24 weeks gestation. The myotube fiber is found before 12 weeks gestation (Dubowitz, $1963,1965,1966)$. The myoblasts are found round these myotube. These structures reflect the large size of the CSA of muscle fiber at early stage. Comparing superior with inferior hyoid muscles, the CSA of muscle fiber in inferior hyoid muscle, the SO, the IO, and the STH are large at 12 weeks. The muscle fiber of the inferior hyoid muscles can be undeveloped compared with superior hyoid muscles in these embryonic stages. In general the CSA of muscle fiber increased during development, and is only found in the SO, the IO and the TH from 16 weeks. Most hyoid muscles displayed various levels of the CSA in muscle fiber. 
Function in hyoid muscle during development

Ohtani et al. (1988) reported that the endomysium of the dog lingual muscle was composed of two types of fibers which were separated by different arrangements of collagen fibers. Helically arranged collagen fibrils surrounding muscle fibers act to resist stretch forces along the muscle fibers (Schmalbrauch, 1974). Bundles of collagen fibrils allow freedom of motion of individual muscle fibers (Fawcett, 1986). Therefore, collagen fibrils in each muscle play different roles in movement. In particular, the ensheaths of the endomysium are relatively extensible in longitudinal directions. The mechanical strength of the endomysium of hyoid muscles increased during development when covered by fine complex fibers of the endomysium. Therefore, the endomysial ensheaths displayed provided reinforcement for their movements. In particular, a large number of collagen fibers and complex networks were found at postal stage during development. Fawcett (1986) reported that collagen fibers play a role in the freedom of motion of individual muscle fibers during movement. The endomysium is composed of helically arranged and longitudinal bundles of collagen fibrils (Plenk, 1927, 1934; Ushiki and Ide, 1986; Ohtani et al., 1988), which are stained in silver-impregnated preparations (Laidlaw, 1930; Nageotte, 1932).

\section{Development of reticular fiber}

The two types of fibers are separated by different arrangements of collagen fibers in the endomysium (Ohtani et al., 1988). Sato and Shimada (1997) found collagen fibers changed in volume and form at each stage in the development of postnatal rat masticatory muscle. In the first stage thin fibers formed irregularly arranged networks; in the second stage fibers increased in number. In the third stage, collagen bundles formed. In the four stage, these bundles increased in number, and in the fifth stage bundles became the thickest in development of the muscle fibers. In our results, although reticular fiber increased gradually, the CSA of muscle fiber did not show same profiles. The percentage of reticular fiber was high in value, in contrast with the low value of the CSA of muscle fiber at 20 weeks in inferior hyoid muscle; the IO, the STH, and the ST and the superior muscle; the MH. Purslow (1994) demonstrated reorientation of collagen fibrils in the endomysium with changing muscle length. During development the intramuscular connective tissue changed the form and the contents in each hyoid muscle associated with increasingly dense reticular fiber. Moreover, the specific profiles are associated with extracellular matrices. The connective tissue network of the epimysium, perimysium and endo- mysium in the skeletal muscle is mainly composed of collagenous protein such as collagen types I and III (Mayne and Sanderson, 1985). Robinson et al. (1988) also reported that endomysium contained collagen types I and III in cardiac muscle. Other extracellular matrices affect cell morphology and the cytoskeletal organization of actin microfilaments (Kreis and Vale, 1993). Therefore, the morphology and the migration of the reticular fiber at each stage reflect the intensity of immunoreactions of proteins.

\section{References}

1) Birnholz JC, Stephens JC and Faria M. Fetal movement patterns: a possible means of defining neurologic developmental milestones in utero. Am J Roentgenol 1978; 130:537-540.

2) Chevallier A, Kieny $M$ and Mauger A. Limb-somite relationships: origin of the limb musculature. J Embryol Exp Morphol 1977; 41:245-258.

3) Dubowitz V. Enzyme maturation of skeletal muscle. Nature 1963; 197:1215.

4) Dubowitz V. Enzyme hystochemistory of skeletal muscle, Part 2: Developing human muscle. J Neurol Neurosurg Psychiatry 1965; 28:519-524.

5) Dubowitz V. Hystochemistory, enzyme hystochemistory of developing human muscle. Nature 1966; 211:884-885.

6) Fawcett DW. A Textbook of Histology. W.B. Saunders, Philadelphia, 1986.

7) Hooker D. The Prenatal Origin of Behavior, Univ of Kansas Press, pp 1-200, Lawrence, Kansas, 1952.

8) Humphrey $T$. The development of mouth opening and related reflexes involving the oral area of human fetuses. Ala J Med Sci 1968; 5:126-157.

9) Kieny $M$ and Chevallier A. Existe-t-il une relation spatiale entre le niveau d'origine des cellules somitiques myogènes et leur localisation terminale dans l'aile? Arch Anat Microsocp Morph Exp 1980; 69:35-46.

10) Kreis T and Vale R. Guidebook to the Extracellular Matrix and Adhesion Proteins, Oxford University Press, pp 32-66, Oxford, 1993.

11) Laidlaw GH. Silver staining of the endoneurial fibers of the cerebrospinal nerves. Amer J Pathol 1930; 6:435-444.

12) Mayne $R$ and Sanderson $R D$. The extracellular matrix of skeletal muscle. Collagen Rel Res 1985; 5:449-468.

13) McLennan IS. Differentiation of muscle fiber types in the chicken hindlimb. Dev Biol 1983; 97:222-228.

14) McLennan IS. Localization of transforming growth factor beta 1 in developing muscle: implications for connective tissue and fiber type pattern formation. Dev Dynam 1993; 197:281-290.

15) Nageotte J. Sheaths of the peripheral nerves. Nerve degeneration and regeneration; In Pen W (ed): Cytology and Cellular Pathology of the Nervous System. pp 189-239, Hoeber, New York, 1932.

16) Narusawa M, Fitzsimons RB, Izumo S, Nadal-Ginard B, Rubinstein NA and Kelly AM. Slow myosin in developing rat skeletal muscle. J Cell Biol 1987; 104:447-459.

17) Nishimura T, Ojima K, Liu A, Hattori A and Takahashi K. Structural changes in the intramuscular connective tissue during development of bovine semitendinosus muscle. Tissue \& Cell 1996; 28:527-536.

18) Ohtani O, Ushiki T, Taguchi T and Kikuta A. Collagen fibrillar networks as skeletal frameworks: a Demonstration 
by cell-maceration/scanning electron microscope method. Arch Histol Cytol 1988; 51:249-261.

19) Plenk H. Über argyrophile Fasern (Gitterfasern) und ihre Bildungszellen. Ergebn Anat Entw Gesch 1927; 27:302412.

20) Plenk H. Die Schannsche Scheide der markhaltigen Nervenfasern. Z Mikrosk Anat Forsch 1934; 36:191-214.

21) Purslow PP and Trotter JA. The morphology and mechanical properties of endomysium in series-fibered muscles: variations with muscle length. J Mus Res Cell Motil 1994; 15:299-308.

22) Rizk NN. Stereoscopic visualization of various morphological types of collagenous fibers. Acta Anat 1980; 107:424-429.

23) Robinson TF, Cohen-Gould L and Stephen Factor. Structure and function of connective tissue in cardiac muscle: collagen types $I$ and III in endomysial struts and pericellular fibers. Scan Micro 1988; 2:1005-1015.

24) Sano $Y$. Über die Neurosekretion der Vorderhornzellen im Lumbalmark der Vogel. Folia anato Jap 1954; 26:1-9.

25) Sato I and Shimada K. Postnatal ultrastructural changes in the perimysium and endomysium of the masticatory muscles in the rat; In Motta PM (ed): Recent Advances in Microscopy of Cells, Tissues and Organs, Kluwer Academic Publishers, pp. 117-183, Boston, 1997.
26) Schmalbruch $H$. The sarcolemma of skeletal muscle fibers as demonstrated by a replica technique. Cell Tissue Res 1974; 150:377-387.

27) Streeter GL. Weight, sitting height, head size, foot length, and menstrual age of the human embryos. Contrib Embryol 1920; 55:146-169.

28) Streeter GL. Developmental horizons in human embryos, description of age group XI, 13 to 20 somites and age group XII, 21 to 29 somites. Contrib Embryol 1942; 197:213-245.

29) Streeter GL. Developmental horizons in human embryos, description of age groups XV, XVI, XVII, and XVIII. Contrib Embryol 1948; 211:135-203.

30) Streeter GL. Developmental horizons in human embryos, description of age groups XIX, XX, XXI, XXII, and XXIII. Contrib Embryol 1951; 230:167-196.

31) Ushiki $\mathbf{T}$ and Ide $\mathbf{C}$. Three-dimensional architecture of the endoneurium with special reference to the collagen fibril arrangement in relation to nerve fibers. Arch Histol Jpn 1986; 49:553-563.

32) Vries JIP, Visser GHA and Prechtl HFR. The emergence of fetal behavior. I. Qualitative aspects. Early Human Develop 1982; 7:301-322.

33) Walls EW. The microanatomy of muscle. In Bourene GH (ed): Structure and Function of Muscle, Academic Press, Vol. 6, pp 21-61, New York and London, 1960. 
Plate I

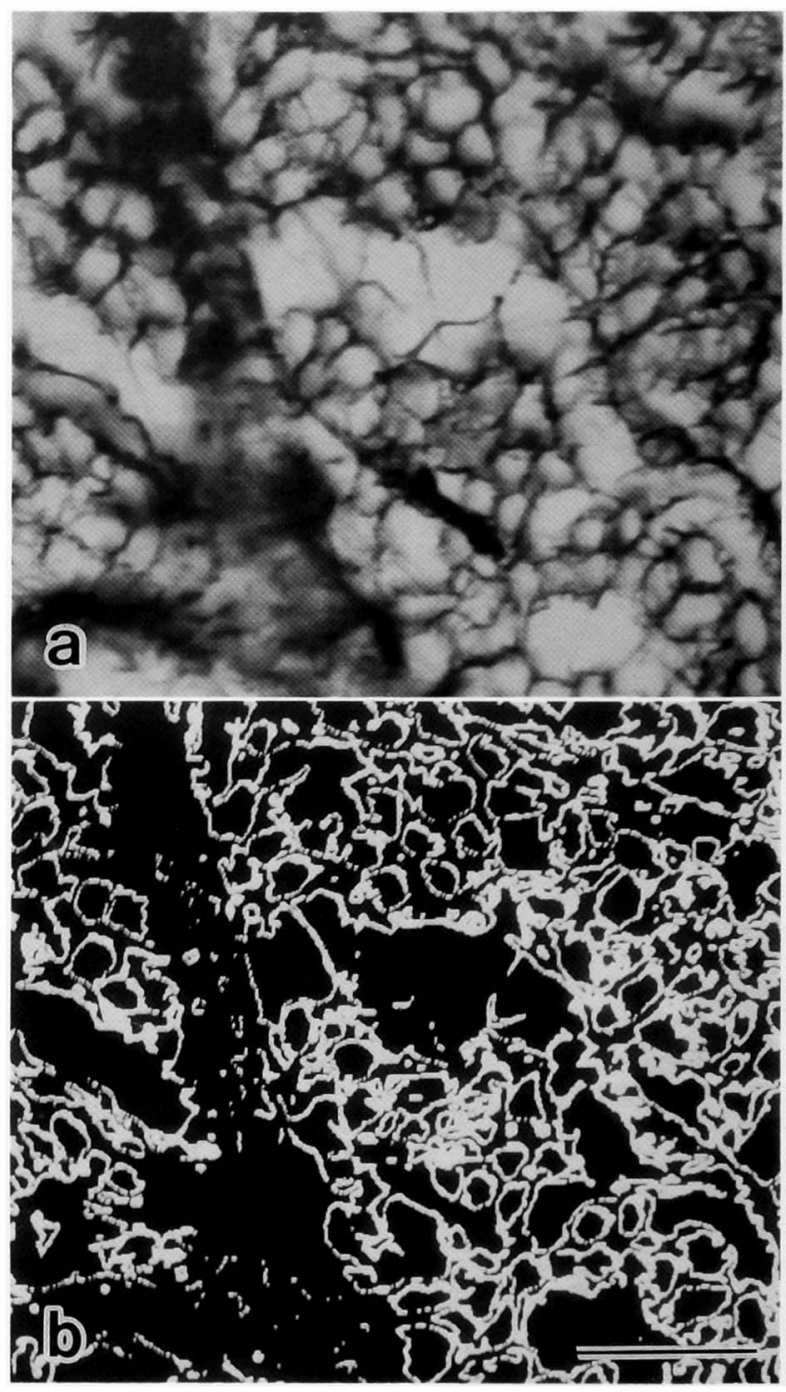

\section{Explanation of Figures}

\section{Plate I}

Fig. 1. Densitometric profile of the endomysium of the cross sections of muscle fibers in the human anterior belly of digastric with a silver impregnation technique determined with a color image analyzer linked to a microcomputer. a, Color photograph with endomysium (black). b, Image obtained determined by computer analysis (bar $=100 \mu \mathrm{m})$. 
Plate II

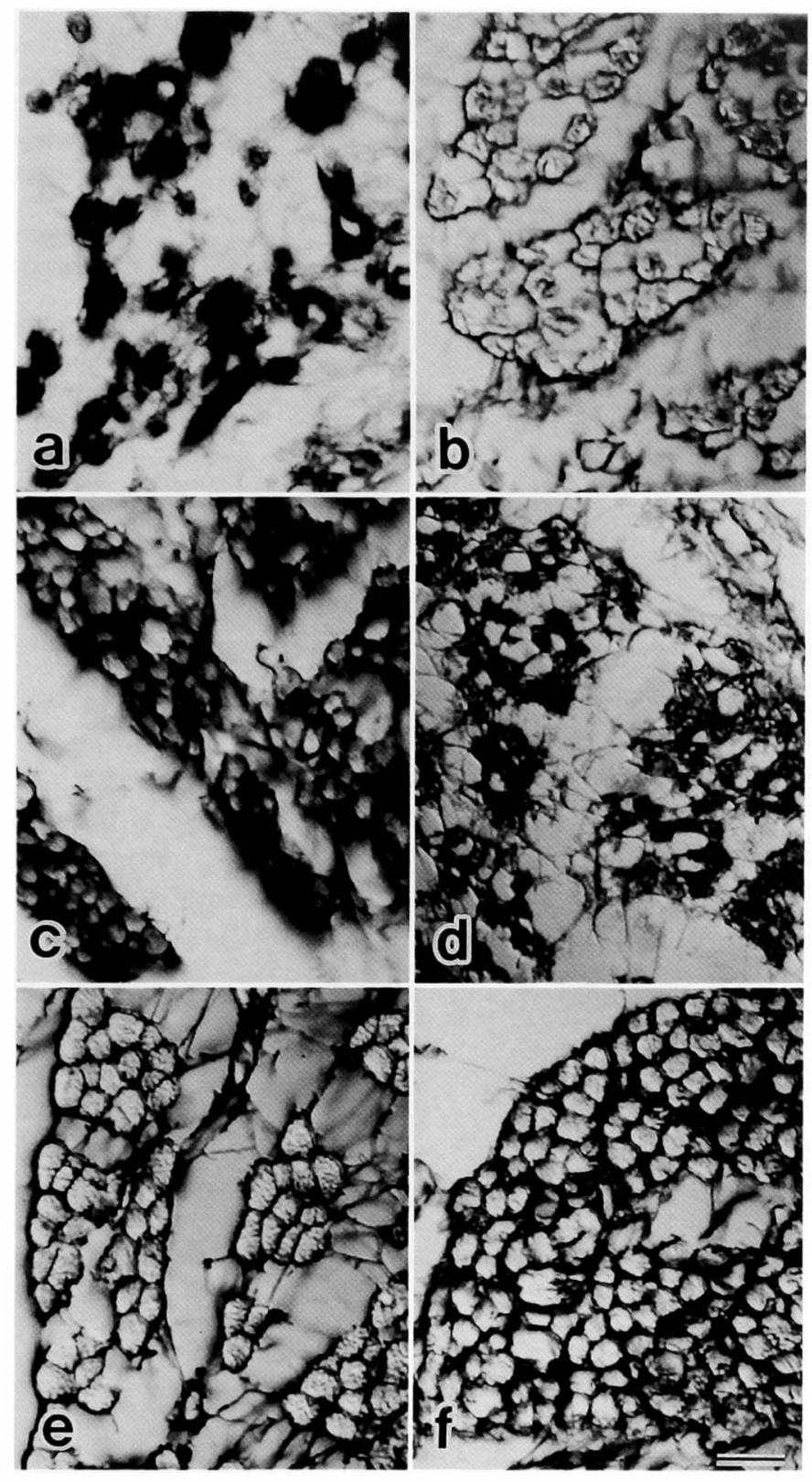

\section{Plate II}

Fig. 2. Light micrographs of cross sections of muscle fibers in the human anterior belly of digastric with a silver impregnation technique. a, At 12 weeks gestation, the endomysium is composed of complex structures near the muscle fibers. b, At 16 weeks gestation, the endomysium is composed of irregularly arranged fibers. c, At 20 weeks gestation, the endomysium forms a complex network structure, with fine fibers between each muscle fiber. d, At 24 weeks gestation, the endomysium is composed of large bundles arranged. e, At 28 weeks gestation, the endomysium is composed a complex network structure. f, At 32 weeks gestation, the endomysium is arranged regularly $(\mathrm{Bar}=100 \mu \mathrm{m})$. 

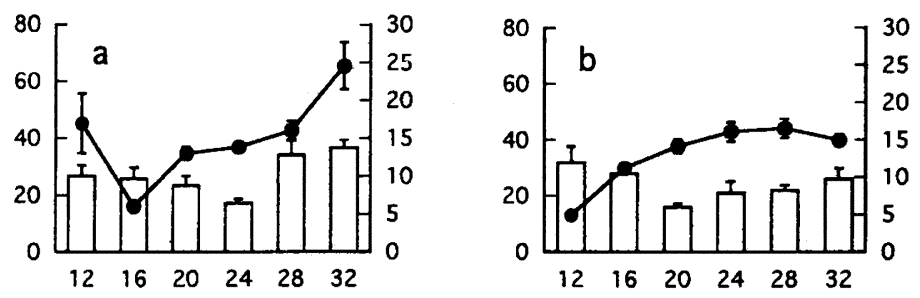

Plate III
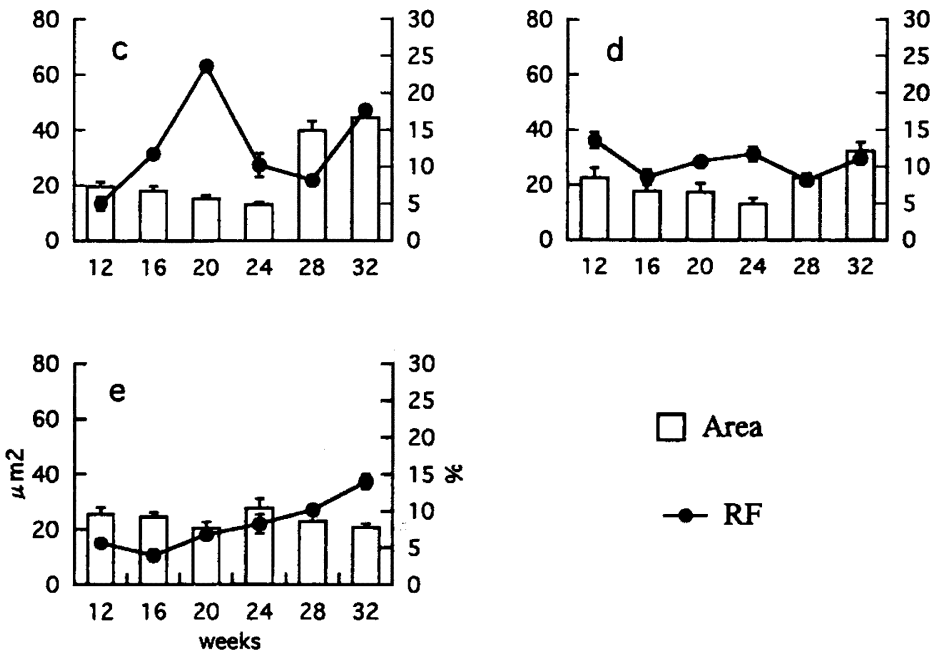

Area

$\rightarrow$ RF

\section{Plate III}

Fig. 3.a. Changes of percentage of reticular fiber (RF) in the intramuscular connective tissue using a silver staining and the cross sectional area of muscle fiber in the superior hyoid muscles. $a$, anterior belly of digastric; $b$, posterior belly of digastric; c, mylohyoid; d, stylohyoid; e, geniohyoid
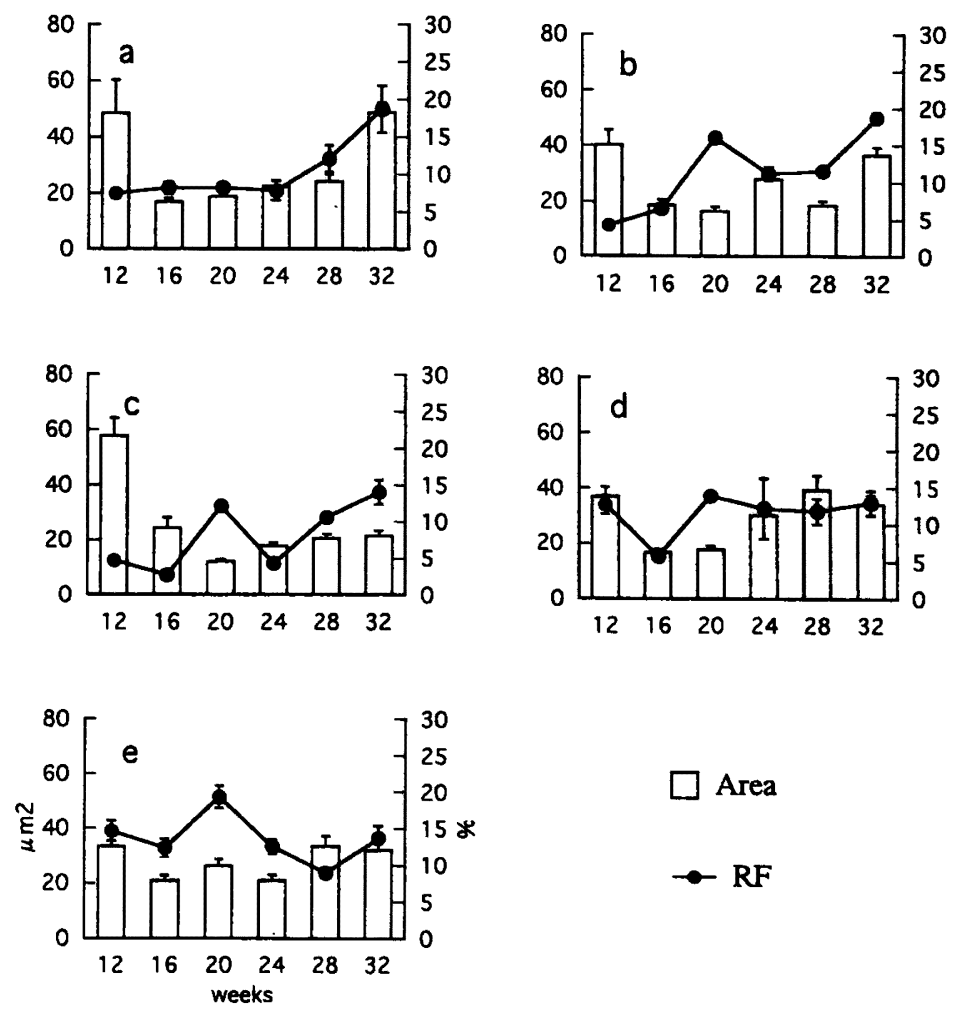

Area

RF

Fig. 3.b. Changes of percentage of reticular fiber (RF) in the intramuscular connective tissue using a silver staining and the cross sectional area of muscle fiber in the inferior hyoid muscles. a, superior belly of omohyoid; $b$, inferior belly of omohyoid; c, sternohyoid; d, thyrohyoid; e, sternothyroid 